\title{
Tabvertising: nuevas fórmulas publicitarias en las tabletas digitales
}

\author{
Sandra MARTÍNEZ COSTA ${ }^{1}$ \\ Natalia QUINTAS FROUFE ${ }^{2}$ \\ Antonio SANJUÁN ${ }^{3}$ \\ Universidade da Coruña
}

\begin{abstract}
RESUMEN:
En este artículo se plantea una definición de los formatos publicitarios más frecuentemente utilizados en las tabletas digitales tipo iPad. El objetivo es el de proporcionar al anunciante un panorama global que le permita entender mejor qué tipo de publicidad hacer para alcanzar a su público objetivo a través de estos soportes.
\end{abstract}

PALABRAS CLAVE: Tabletas digitales; publicidad; apps; tabvertising

TITLE: Tabvertising: New advertising forms on digital tablets

\section{ABSTRACT:}

This article proposes a definition of advertising formats most frequently used on digital tablets as iPad, for example. The aim is to provide advertisers an overview that allows a better understanding about which type of those advertising reaches best their target.

KEY WORDS: Digital tablets; advertising; apps; tabvertising

\section{Introducción}

En las últimas décadas los medios digitales han ido revolucionando las formas de comunicación facilitando su manifestación, bien sea esta social o interpersonal, en cualquier momento y lugar y generando fórmulas publicitarias cada vez más eficaces. El boom de las nuevas tecnologías aplicadas a la comunicación hace posible la percepción del "empequeñecimiento del mundo" y la eliminación de los lugares físicos como barreras. La consecuencia es un cambio en el modo, la forma y la percepción que se establece de la comunicación, que afecta a la creación y difusión de contenidos audiovisuales y publicitarios. Por lo tanto la convergencia con los medios móviles hace necesaria una redefinición de esa esfera espacial y temporal

\footnotetext{
1 E-mail: s.martinez@udc.es

2 E-mail: n.quintas.froufe@udc.es

3 E-mail: sanjuan@udc.es
} 
en la que se transmite la comunicación, creando modelos que integren actividades comunicativas en esas nuevas formas de transmisión.

Los dispositivos móviles, diseñados para ser cada vez más pequeños, rápidos o ligeros, con mayor autonomía y preparados para la comunicación wireless, son también medios de convergencia donde las nuevas tecnologías mezclan la comunicación interpersonal con el entretenimiento y otro tipo de utilidades que puedan ser llevadas a cabo en cualquier momento y lugar. En España la banda ancha móvil ya ha superado con casi 14 millones de líneas a la banda ancha fija ${ }^{4}$. La posibilidad de conectarse a Internet desde casi cualquier parte del mundo hace factible la emisión de contenidos publicitarios personalizados y exclusivos, interactivos, y con posibilidad de retorno en el usuario.

El nacimiento de las tabletas digitales permite un uso más completo del dispositivo móvil, ya que aúna las funcionalidades de un ordenador con la ligereza y autonomía de un teléfono, pero con un nuevo concepto de ergonomía. Además, su pantalla de mayor tamaño y definición hace que sea un soporte mucho más visual y atractivo para los contenidos publicitarios, al permitir una gran definición de imagen y un color mucho más nítido. Y, obviamente, la interactividad supone un valor añadido adicional. Por todo ello, en los últimos años se ha venido generando entre los publicistas y anunciantes un interés creciente por las capacidades de los nuevos medios y soportes digitales como sistemas de difusión publicitaria.

Las múltiples posibilidades técnicas y de experimentación que pueden darse en estos dispositivos permiten enriquecer el mensaje publicitario y aumentar así su atractivo de cara al consumidor. El cambio conceptual y de funcionalidad que suponen las tabletas implica también un nuevo concepto de diseño en lo que al desarrollo de aplicaciones se refiere. No obstante, el impacto, desarrollo y alcance que ofrecen dichos dispositivos todavía no han sido aprovechadas al máximo por los anunciantes. Muchas marcas no han realizado acciones en dichos soportes quizá por el desconocimiento de los beneficios de las mismas, o por la dificultad en el diseño y desarrollo de la aplicación más adecuada para su contenido publicitario. La novedad de estos dispositivos y de sus posibilidades de interactividad hacen necesarias investigaciones que aborden esta nueva realidad desde la perspectiva publicitaria.

Con el fin de definir las tipologías de publicidad más frecuentemente utilizadas en las tabletas digitales, este artículo recoge los primeros resultados de una investigación más amplia que se está desarrollando en la Facultad de Ciencias de la Comunicación de la Universidade de A Coruña sobre las nuevas fórmulas publicitarias y periodísticas en las tabletas digitales.

4 Fundación Telefónica (2012): Informe de la Sociedad de la Información en España 2011. Madrid, Telefónica. 


\section{Diseño de la investigación: objetivos y metodología}

El objetivo principal de esta investigación es recopilar las fórmulas publicitarias creadas para tabletas digitales. Se trata de comenzar a construir un marco teórico a través de las aportaciones propias y de otros autores.

Esta investigación fue diseñada en dos fases. La primera de ellas se concretó en la revisión de la literatura reciente. Se consultaron todas las publicaciones científicas del área de Comunicación recogidas en el IN-RECS con el fin de recopilar la información más reciente acerca del fenómeno estudiado. Se tomó como referencia el período comprendido desde 2010, por ser la fecha de lanzamiento del iPad, hasta la actualidad. En total se analizaron todo los números de las 24 revistas recogidas en la actualización del último IN-RECS (año 2010).

En esa primera fase de recopilación no se encontró ninguna referencia específica sobre la publicidad en las tabletas digitales, muestra evidente de la novedad del fenómeno estudiado. Por el contrario, sí se observó una tendencia hacia el estudio de los videojuegos como soporte publicitario o el advergaming y la publicidad en las redes sociales (véase por ejemplo Telos, $\mathrm{n}^{\circ} 85,2010$ ).

Merece ser destacado el número 28 de la revista Trípodos dedicado a la Neopublicidad, en la que se publican varios textos acerca de la adaptación de la publicidad al entorno digital y a los nuevos formatos utilizados en los móviles. Otra publicación que muestra interés por dicha temática es la revista Sphera pública: revista de ciencias sociales y de la comunicación, con un número específico sobre publicidad (Investigación en publicidad: teoría, transformaciones, medios y público) en la cual se incluyen referencias sobre publicidad en redes sociales e internet.

La segunda fase consistió en localizar, clasificar y analizar los principales formatos publicitarios en las tabletas digitales a través de una muestra aleatoria de más de cien anunciantes con el fin de establecer una clasificación acerca de los más comúnmente utilizados. Para ello se hizo la selección de los más descargados en la categoría de "estilo de vida" en el App Store de Apple. Se escogió este por ser el sistema operativo para tabletas digitales con mayor índice de descargas en España. El trabajo de recopilación y análisis fue llevado a cabo por tres investigadores durante un periodo de cuatro meses, en los que se analizó y se procedió a una clasificación en base a categorías previamente establecidas.

Los datos adquiridos en estas dos fases de la investigación se completarán en una tercera etapa con una revisión del trabajo de los profesionales del sector a través de entrevistas en profundidad. 


\section{Las tabletas digitales en el mercado publicitario actual}

El sector publicitario actual se ha visto afectado por dos hechos que repercuten también en el comportamiento del consumidor: la crisis económica y la saturación publicitaria.

La crisis económica obliga al consumidor a ser más selectivo en sus compras; necesita disponer de más información sobre el producto y realiza mayores comparaciones entre opciones similares antes de tomar la decisión final. Dicha crisis también se ha trasladado al mercado publicitario. Según recoge el estudio Infoadex sobre la inversión publicitaria en España en el año 2011 se constata una «tasa de crecimiento negativo del 6,5\% en el año 2011 para el total del mercado, incluyendo medios convencionales y no convencionales $\rangle^{5}$. Dicha tendencia negativa, iniciada en el 2008, hace difícil prever hacia dónde se encaminará el comportamiento del mercado.

Por otra parte, ante la saturación publicitaria, el consumidor rechaza cada vez más aquella de carácter convencional que lo trata como audiencia y opta por tomar la iniciativa buscando la publicidad de su interés. El sector ha reaccionado y por ello la inversión en medios no convencionales superó en 2011 a la de los convencionales $^{6}$. Como reconoce Benavides, la saturación «favorece la búsqueda de nuevos soportes y formas de contacto de las empresas con sus públicos, además de una ruptura e hibridación de los formatos tradicionales a favor del desarrollo de nuevos modos de comunicación al amparo de las tecnologías digitales» ${ }^{7}$.

En este contexto se presentaron las tablets o tabletas digitales en 2010. Estos dispositivos, liderados en la actualidad por el iPad de Apple, consiguieron abrirse un hueco en el mercado y en la mente de los potenciales consumidores. El abaratamiento del hardware y el desarrollo de sistemas operativos diferentes del IOS de Apple multiplicaron el número de aplicaciones y dispositivos que, previsiblemente, dejarán obsoletos en breve a los más tradicionales netbooks y a los e-readers ${ }^{8}$. Prueba de ello es el hecho de que, según datos del segundo trimestre de 2011, las ventas de tablets (cifradas en 13,6 millones) superaron las de los netbooks, $(7,3$ millones) ${ }^{9}$. Además, las previsiones para el mercado de las tabletas son prometedoras, ya que se estima que uno de cada tres consumidores tendrá una en tan solo dos

\footnotetext{
5 SÁnchez Revilla, M. A. (2012): Resumen. Estudio Infoadex de la inversión de la comunicación publicitaria 2011, Madrid, Infoadex S.A.. http://www.infoadex.es/RESUMEN2011.pdf. Web visitada el 25 de febrero de 2012.

6 Ibidem

7 Benavides Delgado, J., (et al.). (2010). «Los anunciantes españoles y el nuevo contexto de comunicación: una aproximación cualitativa», Revista Latina de Comunicación Social, 65, 159-175.

8 Fundación Telefónica (2011): Informe de la Sociedad de la Información en España 2010. Madrid, Telefónica.

9 Fundación Telefónica (2012): Informe de la Sociedad de la Información en España 2011. Madrid, Telefónica.
} 
años más ${ }^{10}$. Como consecuencia de ello, se prevé un incremento de la inversión publicitaria en este tipo de soportes y por lo tanto de una mayor competitividad entre marcas.

El informe Distimo ${ }^{11}$ recoge los últimos datos de aplicaciones descargadas según categorías. Las dedicadas a la información del tiempo, quiosco y entretenimiento encabezan la clasificación. En cuanto al uso de las aplicaciones relacionadas con marcas, el $\mathrm{IAB}^{12}$ publicó en noviembre de 2011 un estudio que indicaba que los usuarios admitieron utilizarlas fundamentalmente para el ocio, mientras un $55 \%$ de los usuarios dijeron utilizarlas como herramienta de trabajo. En el mismo estudio del IAB se analizó el tipo de uso de las aplicaciones asociadas a marcas. Fundamentalmente, el usuario las usa para buscar lugares de interés (52\%), utilizar la geolocalización (51\%), consultar información de empresas de las que son clientes $(50 \%)$ y adquirir información relacionada con viajes (40\%) así como apps de moda $(23 \%)$ y outlets $(15 \%)$. El hecho de que en esta fase inicial las tabletas estén asociadas a "estar a la última" hace pensar que sus usuarios tienden al consumo de productos de moda y tendencias, con lo que las aplicaciones relacionadas con este campo pueden tener un alto número de descargas entre ellos.

Las tabletas digitales han revolucionado la ergonomía de uso de los dispositivos móviles, ya que no necesitan de elementos externos como teclados o ratones y permiten una postura más relajada. No tiene ataduras de cables y su autonomía es muy alta, dado que su consumo energético es mínimo y permite su uso durante 6-8 horas sin necesidad de recarga.

Otro dato relevante es que la mayoría de estos soportes son accesibles a cualquier tipo de consumidor y no necesariamente a los más altos tecnológicamente hablando. Su navegación es sencilla e intuitiva, pensada para un usuario medio sin pretensiones tecnológicas, lo que lo hace potencialmente asequible a todo tipo de públicos objetivos. Esto no solo condiciona su tipo de uso sino también el tipo de aplicaciones que se desarrollan para él. El tiempo de navegación es mayor que con un ordenador, ya que la postura es más cómoda, y el tamaño de la pantalla y la navegación completamente táctil las hacen más agradables de usar que los teléfonos móviles ${ }^{13}$.

La versatilidad de estos dispositivos, su ergonomía novedosa y de fácil adaptación a varios tipos de tareas hace que sus usos sean múltiples. Y dado que se trata de un dispositivo ligado al ocio, puede resultar una herramienta publicitaria idónea ya que muchas de las compras se realizan en estos momentos de relax del consu-

10 Puro Marketing. http://www.puromarketing.com/12/11392/cada-tres-consumidores-lineautilizara-tablet-2014.html\%29. Web visitada el 25 de diciembre de 2011.

11 Distimo. http://www.distimo.com/. Web visitada el 25 de febrero de 2012.

12 IAB Spain. III Estudio IAB Spain sobre Mobile Marketing. http://www.iabspain.net/. Web visitada el 10 de diciembre de 2011.

13 Budiu, R. \& Nielsen, J. (2011). «Usability of iPad Apps and Websites» 2nd Edition, Publisher: Nielsen Norman Group, 1-129. 
midor. Además, la fiabilidad en la adquisición de productos a través de estos dispositivos cada vez es mayor.

Sin embargo, el por ahora elevado precio de las tabletas puede ser considerado una barrera a la hora de adquirir el dispositivo. El público no lo estima como un elemento necesario e imprescindible sino más bien de lujo, por lo que su adopción puede ser más lenta hasta que se normalice su adquisición.

\section{Modelos de publicidad para tabletas digitales: Tabvertising}

Esta nueva fórmula publicitaria, surgida de la combinación de los términos tablet + advertising, parece irrumpir con fuerza en la publicidad actualmente. A la espera de que la estabilización en el mercado de las tabletas digitales genere formas de comunicación y formatos publicitarios más complejos, se puede hacer una clasificación de los más frecuentemente utilizados hasta el momento en este tipo de soportes. La tipología propuesta parte de la observación de dichos formatos y únicamente pretende una aproximación a este fenómeno ya que todavía no existe ninguna clasificación unificada al respecto.

Esta tipología se basa, fundamentalmente en dos clases de formatos publicitarios: los derivados de prensa y web y los creados de forma específica y original como aplicaciones publicitarias por el propio anunciante. En el primero de los grupos se incluyen los siguientes:

1. Formatos publicitarios tradicionales derivados de la prensa: página completa, robapáginas, faldones, rascacielos, etc. interactivos o no y que pueden estar enriquecidos con contenido de vídeo y audio. Son los que se incluyen en publicaciones editoriales, como revistas o periódicos y en la mayor parte de las ocasiones se trata de los mismos anuncios que en el soporte papel.

2. Formatos publicitarios derivados de la web: generalmente banners, pop-ups, etc. Permiten la presencia de pequeños espacios publicitarios cuando el usuario navega o utiliza determinados servicios de su tableta. Entre este grupo de formatos cabe destacar los intersticiales, formato de anuncio a pantalla completa que se abre al cargar una aplicación. Generalmente aprovecha para aparecer en el tiempo de espera del usuario. Debe ser este quien lo cierre aunque en algunos casos desaparece pasados unos segundos.

En cuanto a los formatos publicitarios generados por el propio anunciante originalmente para tabletas digitales, y basados en la estructura de una app para iOS o Android, podrían incluirse los siguientes:

1. Aplicaciones patrocinadas por la marca con contenidos relacionados con ella, que aportan un valor de uso independiente para el consumidor. Algunos ejemplos de ello serían Sopalista, Nike Training, Pirelli Diablo, etc. La finalidad no 
es la de ser un soporte meramente publicitario sino la de proporcionar al usuario una experiencia de uso diferente a la de la simple navegación-compra de los productos de la marca y generar así fidelización.

2. Catálogos de visionado de productos: Ikea, H\&M, iBeetle, Mini Getaway, etc. El objetivo es el de centrarse en el producto publicitado y que la experiencia del usuario sea básicamente la del conocimiento de la marca.

3. Juegos: El advergaming y el advertainment, mezcla de entretenimiento y publicidad, es un medio eficaz para dar a conocer marcas y fidelizar al usuario. La descarga de juegos promocionales de películas o de determinadas marcas publicitarias hace que en este caso publicidad y entretenimiento se encuentren fusionados. La gran campaña de lanzamiento que «Los Pitufos» hizo con el estreno de la película, y la posibilidad de descarga del juego para iPad y Facebook, hizo posible un conocimiento más detallado del argumento del film. Por otro lado, el propio patrocinio y el product placement en los juegos, supone otro nuevo campo de integración para la publicidad. Con esta modalidad se pretende generar en el usuario una experiencia de uso y entretenimiento que va más allá de la marca y que busca, también, la fidelización del cliente a través del ocio.

4. Aplicaciones de servicios: Pueden estar basados en la geolocalización, en el contacto directo con el usuario, etc. Este sería el caso de, por ejemplo, Metros de Madrid y Barcelona y servicios de autobuses de las distintas ciudades. En este caso la finalidad es más utilitaria, pero no obstante debe tenerse en cuenta que en muchas ocasiones los servicios están proporcionados por empresas privadas.

5. Publicaciones digitales de las distintas marcas. Se trata de revistas promocionales de las marcas con contenidos multimedia que podrían ser o no interactivos y en un formato similar a la tradicional revista de comunicación de las empresas. Sin embargo, en muchos casos la marca anunciante incorpora a este formato publicitario contenido al margen de la marca de interés para el usuario (turístico, entrevistas, etc.). Son ejemplos de ello Audi Magazine, Nike 1948 o Ferrari Mag. entre otros.

Cabe destacar también que en el transcurso de la redacción de este artículo Google anunciaba el lanzamiento de formatos publicitarios específicos para tabletas (todavía en beta). Falta por comprobar si estos se adaptarán a los ya existentes y resultarán una "forma automatizada" de creación de contenidos comerciales, o si se generarán nuevos modelos distintos de los ya existentes en las stores de Android y Apple.

En definitiva, si tenemos en cuenta lo dicho hasta el momento podemos intuir que las estrategias futuras se reorientarán hacia aquellas consideradas como pull en las que «los individuos tiran del formato y por ende del mensaje publicitario que en 
él anide bajo alguna de sus camaleónicas acepciones» ${ }^{14}$. Deja constancia de ello el hecho de que una gran parte de los contenidos publicitarios están relacionados con apps, implicando una actitud de búsqueda y aceptación por parte del consumidor. De esta forma, el concepto on demand toma gran relevancia en los contenidos publicitarios.

Todavía es pronto para valorar la eficacia de dichos modelos publicitarios por la novedad que supone el propio dispositivo para el consumidor y para la propia industria, y sobre todo es difícil medir cuál es el tipo de aplicación y de contenido útil para cada usuario. La tendencia es a un uso cada vez mayor de modelos publicitarios no intrusivos, por ello las agencias y anunciantes se esfuerzan por solicitar la autorización a los clientes potenciales para la recepción de mensajes comerciales. De esta manera, no sólo se consigue la aceptación del público hacia la campaña y la recepción de publicidad, sino también sus datos personales e incluso en algunos casos, datos sobre gustos y preferencias lo que permite perfilar en mayor medida el público objetivo del mensaje. De este modo se consigue que el consumidor no se sienta invadido en su espacio personal y que tenga un mínimo interés por la campaña que está recibiendo a través de diferentes soportes móviles.

\subsection{La experiencia interactiva del usuario en el contexto publicitario actual}

El usuario/público objetivo de este nuevo modelo de publicidad no responde a los patrones tradicionales de comportamiento sino que se muestra con un nuevo perfil que trataremos de definir:

1) Es un usuario eminentemente activo y dinámico y ello provoca un feedback que el anunciante puede recibir en prácticamente tiempo real dando lugar a una interacción inmediata entre consumidor/anunciante. El consumidor, o el ya denominado por muchos prosumidor, puede interactuar con la marca e implicarse más en el mensaje que ésta genere e incluso colaborar en su creación. Como ha resumido Corredor, «se trata del final del discurso unidireccional de las marcas y la inauguración de una era donde el consumidor asume el protagonismo de la comunicación comercial y ejerce su poder e influencia en la Red» ${ }^{15}$. En esa misma línea, De Salas afirma que «es la era del Marketing UNO a UNO, donde la relevancia de la información es más importante que nunca al poder elegir el usuario la publicidad que recibe» ${ }^{16}$.

14 Del Pino, C. (2007). «Nueva era en la comunicación comercial audiovisual: el marketing viral», Pensar la Publicidad, vol. I (2), 74.

${ }_{15}$ Corredor Lanas, P. (2011). «En clave digital: nuevos profesionales en Publicidad», Telos, 87.

16 De Salas Nestares, Mª I. (2009). «La publicidad en las redes sociales: de lo invasivo a lo consentido», Icono $14,15,81$. 
La posibilidad de una vía de retorno se presenta como fundamental. El soporte no es solo una plataforma de difusión publicitaria, sino también una vía de comunicación con el cliente, bien unidireccional, bien multidireccional entre los propios consumidores y la marca. Esto anima a tener un control activo sobre el mensaje publicitario tomando un determinado número de decisiones. Esta interactividad debe ser además lo suficientemente eficaz y de calidad, como para que se genere satisfacción y fidelización entre el público. Los servicios llenos de contenido, crean en el espectador una satisfacción mayor que la de la publicidad tradicional. Actividades tales como compras online, visionado y descarga de spots, catálogos, juegos etc., permitirán al espectador/consumidor un mayor control sobre el mensaje publicitario.

2) Es un usuario/consumidor always on. Un consumidor que se conecta desde su tableta a internet todos los días en el 80 por ciento de los $\operatorname{casos}^{17}$.

3) Como consecuencia de lo anterior, el usuario puede compartir. Por tanto la potencial viralidad del mensaje cobra más relevancia si cabe. Cualquier usuario puede «compartir» con su círculo más cercano o su red de amigos algunos de los mensajes publicitarios que considere adecuados; pudiendo hacer lo mismo con las quejas $\mathrm{u}$ opiniones negativas de la marca.

4) Es un consumidor que valora sobremanera la inmediatez, la rapidez y la instantaneidad. También considera que la tableta, así como los smartphones, son instrumentos definidores de su personalidad, en especial a través de las aplicaciones que éste ha descargado e instalado en su dispositivo. Por eso es frecuente ver que los usuarios "presumen" de ellas y las recomiendan a otros.

5) Es un usuario aún por definir. El uso que éste hará de las tabletas es por el momento mayoritariamente de ocio, pero es previsible que estos usos se transformen hacia otros más complejos en los que la información, las redes sociales y los usos profesionales adquieran un mayor peso.

6) La predisposición positiva hacia el mensaje publicitario es mayor, dado que es él mismo quién lo ha buscado. Si el contenido publicitario es interesante, el usuario lo descargará y lo llevará instalado en su dispositivo, «así, la publicidad pasa de interrumpir a sus consumidores a invitarlos a pasar un rato de ocio con la marca, hecho que implica que ambas partes resultan beneficiadas con el intercambio» ${ }^{18}$. La fidelización del cliente, a través de su propia experiencia hace que se generen unos vínculos de confianza que permiten a la empresa almacenar información de una riqueza cada vez mayor. Pero ello también implica que el consumidor pueda rechazar o no elegir el contenido publicitario si no le interesa, con lo que la adecuación público-contenido es fundamental.

17 IAB Spain. III Estudio IAB Spain sobre Mobile Marketing. http://www.iabspain.net/ . Web visitada el 10 de diciembre de 2011.

18 Domingo, G. \& MARTorell, C. (2011). «Una mirada al consumidor de hoy (y de mañana) desde la publicidad», Trípodos, 28, 15. 
Dichas características describen al nuevo perfil de usuario, denominado como «comunicador digital permanente» ${ }^{19}$. A la vista de estos rasgos es evidente que la experiencia interactiva tenderá a enriquecerse cada vez más.

No obstante, todavía queda mucho por decir respecto a la experiencia y la relación del usuario con el propio dispositivo ya que, como señala el informe IAB, «aún se está definiendo su función dentro del universo tecnológico del consumidor» ${ }^{20}$.

\section{Posibilidades para el anunciante}

El anunciante es consciente de que por ahora el índice de penetración de estos dispositivos en España no es muy elevado, pero dado el progresivo abaratamiento en la producción de los mismos en países como la India y el incremento de sus prestaciones, las previsiones de crecimiento exponencial de ventas de tabletas es muy alto. Prueba de ellos es la mayor presencia de dispositivos como el KindleFire de Amazon o la GalaxyTab de Samsung en la cotidianidad de los consumidores.

La difusión de publicidad en estos dispositivos hace que el alcance del mensaje se amplíe suponiendo al anunciante una inversión mínima debido a las posibilidades técnicas. Por ello, algunas de las campañas de publicidad de mayor impacto de los últimos años han seguido una clara tendencia a la convergencia de medios y a la explotación de los soportes móviles como nuevos escaparates publicitarios. La facilidad técnica para el desarrollo de aplicaciones iOS o Android hace que el anunciante ya no tenga que desembolsar un alto precio, tal y como sucede con la difusión en prensa, radio o televisión, medios en los que debe pagar además de por la creación del contenido, por el espacio publicitario. La gran ventaja es, por lo tanto, que no es necesario ampliar demasiado la estructura comunicativa del mensaje. A éste se le añade contenido interactivo $\mathrm{y} / \mathrm{o}$ enriquecido, y de esta forma se adapta al nuevo dispositivo. Sin embargo esta práctica, relativamente habitual en la publicidad para móviles, se está transformando gracias a la entrada en el mercado de nuevos terminales con una mayor calidad técnica, entre ellos las tabletas digitales. Consideramos que la tabvertising resulta sugerente al anunciante por varias razones:

1. Permite la personalización del mensaje y la adaptación del mismo a los gustos y preferencias del consumidor. Nos encontramos con un consumidor que recibe miles de impactos diarios y que cada vez es más difícil captar su atención, por ello «el usuario no sólo quiere una mayor selección de la publicidad que recibe,

19 Fundación Telefónica (2012): Informe de la Sociedad de la Información en España 2011. Madrid, Telefónica.

20 IAB Spain. III Estudio IAB Spain sobre Mobile Marketing. http://www.iabspain.net/. Web visitada el 10 de diciembre de 2011. 
sino que pretende recibir exclusivamente los anuncios que le interesan» ${ }^{21}$. Incluso permite que sea el propio consumidor el que personalice la publicidad en función de sus propios intereses. Podría decirse que prácticamente es un mensaje one to one.

2. Es interactivo en tiempo real, entendiendo como experiencia interactiva aquella en la que el usuario desarrolla una acción de forma libre con una o varias personas o puede modificar los contenidos a su gusto. Es importante que se cree una fidelización y para ello es imprescindible que la experiencia interactiva sea interiorizada por el usuario, que la repetirá en caso de haberse quedado satisfecho en la primera de las ocasiones. La fidelización del cliente dependerá también del tipo de contenido publicitario y del nivel de interactividad que haya tenido.

3. Permite la medición en tiempo real del impacto de la campaña.

4. Las limitaciones de calidad con el visionado de las pantallas o de reproducción de vídeos han desaparecido en este tipo de dispositivos. Actualmente las tabletas digitales cuentan con mejores pantallas que las de los teléfonos móviles, permitiendo el visionado de productos de alta calidad estética y gran definición de imagen. De esta forma, las tabletas resultan dispositivos de alto interés para el anunciante, ya que mejoran la posibilidad de navegación y el diseño de los contenidos, así como la definición de la imagen fotográfica y de reproducción de vídeo. Además, al tratarse de dispositivos nuevos permiten un alto grado de innovación. La publicidad para este tipo de dispositivos móviles sugiere múltiples posibilidades, a nivel técnico y creativo, potencialmente inalcanzables en otro tipo de soportes.

5. La geolocalización permite emplazar al consumidor esté dónde esté y enviarle publicidad específica local de la zona en la que se encuentre, lo que implica una alta personalización del mensaje publicitario. Además son soportes digitales de fácil uso y manejabilidad, de tal manera que permiten su traslado a cualquier lugar sin dificultad. Su autonomía de consumo es también una ventaja.

6. La tecnología wireless permite la descarga de contenidos y la conexión a Internet, facilitando la actualización constante del mensaje y la interacción con el público objetivo.

Pero para que esta nueva fórmula funcione es importante tener en cuenta varios aspectos:

1. El contenido ofrecido debe ser exclusivo: Las dificultades de adaptación del usuario a las nuevas tecnologías digitales hacen que el consumidor busque en ellos contenidos que ofrezcan un valor añadido de exclusividad. La navegación por Internet es más sencilla y cómoda en un ordenador que en un tablet, lo que

21 LÓPEZ, D. (2011). «El digital out of home (dooh) o digital signage, el otro marketing digital», Trípodos, 28, 26. 
implica que la conexión en este soporte debe reportarle otro tipo de beneficios añadidos, bien sea la inmediatez del mensaje, o bien el acceso a contenidos diferenciados.

2. Debe ser práctico: El producto publicitario para soportes móviles debe ser fácilmente manejable, ya que un uso complejo, puede provocar en el consumidor rechazo y renuncia al mensaje. La interactividad, en caso de existir, debe ser lo más sencilla posible, y al mismo tiempo útil, de tal manera que el usuario perciba un servicio añadido que no encontrará en otros soportes.

3. Debe tener unos objetivos claros y definidos: Entre los objetivos a destacar nos encontramos fundamentalmente con que los anunciantes buscan, por un lado incrementar las ventas o beneficios; y por otro, mejorar la relación anunciantecliente. Muchas marcas buscan la fidelización a través de dispositivos móviles.

Por otra parte, el tipo de público de las campañas publicitarias creadas para estos soportes móviles también está variando. Si antes las campañas para móviles se destinaban fundamentalmente a un público joven, la consolidación de la tecnología hace que los anunciantes estén buscando un nuevo tipo de audiencia a la que dirigirse. Es cierto que la i-generación parece ser la audiencia más predispuesta a la aceptación de este tipo de mensajes por su familiaridad con estos dispositivos, sin embargo el target de los mismos ha variado. El acceso a Internet en movilidad continúa creciendo y las tabletas se erigen como uno de los principales dispositivos para navegar en las distintas franjas de edad. No obstante, es necesario tener en cuenta todavía la diversidad de públicos y los distintos grados de interacción aprendida que estos tengan con aquellos soportes con los que interactúan.

De todos modos, para que estas nuevas fórmulas publicitarias se consoliden es fundamental que el usuario se sienta "enriquecido", creándose así una fidelización hacia los productos a través de una auténtica experiencia interactiva. Los contenidos que se proporcionan al usuario, sean estos publicitarios o no, deben responder a experiencias creativas, en lugar de simples gestos en los que el consumidor siga un clic o un enlace sobre un objeto. La experiencia debe ser generada desde el principio de la concepción de la idea.

El formato digital no se añade, sino que debe ser creado desde el origen de la campaña, teniendo en cuenta las especificidades de cada dispositivo. Es frecuente que las marcas generen contenidos publicitarios para el iPhone, por ejemplo, y esa misma aplicación se adapte, con un adecuado escalado de pantalla, a los dispositivos iPad. Pero ello implica un aprovechamiento ineficiente del espacio así como una incorrecta distribución de elementos y botones. En muchos casos la publicidad no gira con el dispositivo puesto que ha sido creado para móviles sin giroscopio o para la web y, en otros, el escalado de la imagen muestra el pixelado de la fotografía utilizada como reclamo publicitario. Además, debe tenerse en cuenta que el entorno «tableta digital» es totalmente distinto en uso y ergonomía de un ordenador y de un móvil, ya que su tamaño incita a pensar más en una postura de «lectura de 
revista». Cuanto mayor sea la interacción ofrecida, mayor es la cantidad de servicios para el consumidor lo que implica que el valor añadido de estos nuevos medios y tecnologías no se encuentra en el incremento del volumen del contenido, ni en la adaptación de la publicidad creada para otros medios, sino en la ampliación de la oferta de servicios.

Por todo ello, uno de los problemas que surge de la interactividad de los nuevos medios es la aplicación y estandarización de los formatos. Trasladar los formatos de Internet no resulta positivo ni viable, ya que la actitud del usuario es distinta. Las diferencias en las pantallas de las tabletas digitales hacen que éste pueda sentirse molesto si la publicidad ocupa una buena parte del espacio del visor. Para que esta fórmula sea útil los contenidos deben ser desarrollados a través de la creatividad. En general, es necesario también un cambio de comportamiento en el consumidor, que deberá adaptarse a las nuevas fórmulas interactivas y aprender pautas de comportamiento distintas de las habituales en la interacción con los medios tradicionales.

En este caso, el anunciante pierde el privilegio de ser el que controle la información emitida, ya que el retorno y la capacidad de interacción se encuentran en manos del consumidor. Esto favorece que las necesidades del usuario sean averiguadas a través de su propia experiencia de uso. Situación que algunos definen como una debilidad del sistema publicitario actual, ya que «los diferentes procesos de tamizado y transformación efectuados por los consumidores pueden poner en serio peligro el concepto original del mensaje, pudiendo conducir a la confusión, mal interpretación o equívoco del mensaje cuando no a su dilución total» ${ }^{22}$.

\section{Conclusiones}

Las fórmulas publicitarias específicas para las tabletas todavía se encuentran en una fase inicial de desarrollo en el contexto español. Estamos en una etapa temprana de la adopción de estos dispositivos en los que se hace difícil extraer conclusiones que no pasen de ser meramente anecdóticas. No obstante, la fuerza con que dichos dispositivos entraron en el mercado no hace más que asegurar un crecimiento en el número de usuarios. Se prevé una mayor tasa de aceptación en los próximos años, que tendrá como consecuencia directa el auge de la tabvertising.

Como se ha visto hasta el momento las posibilidades que presenta esta fórmula publicitaria son muchas pero exige que las marcas y los anunciantes tengan que adaptarse previamente a este nuevo entorno digital. No se trata de trasladar sin más formatos publicitarios a las tabletas digitales sino ver en estos soportes nuevas posibilidades para conseguir sacarles el máximo rendimiento. El anunciante tendrá

22 MARTí PARReÑo, J.M. (et al). (2011). Retos y oportunidades para incrementar la eficacia de la publicidad actual. Comunicación presentada en el III Congreso Internacional Latina de Comunicación Social en Tenerife. 
que integrar también las tabletas en sus estrategias y no simplemente trasladar a ella fórmulas publicitarias típicas de otros medios.

También tendrá que decidir cuál es el formato publicitario de tableta digital más adecuado a sus objetivos y especialmente cuál de ellos funciona mejor entre su público objetivo. Por eso, una vez definidos los modelos más frecuentes es conveniente analizar las posibilidades de cada uno, la propulsión que genera en el usuario y la afectividad o rechazo que provoca el tipo de contenido publicitario.

Y finalmente el anunciante precisará medir el resultado de las acciones publicitarias que propone, evaluar los beneficios que obtiene y valorar las actitudes que los usuarios adopten ante dichos contenidos. Ello exigirá también desarrollar herramientas de análisis más avanzadas que las empleadas hasta el momento.

Todo ello supone una redefinición de las estrategias empleadas, de los mensajes seleccionados y del lenguaje utilizado. Nos encontramos en un nuevo escenario en el que todavía faltan por definir las funciones y (re)conocer las características de los actores principales. Es evidente que el usuario adquiere un papel más relevante en la experiencia interactiva y emerge como una figura más determinante si cabe en el proceso de la comunicación publicitaria. No obstante, es necesario tener un mayor conocimiento de la interacción entre el dispositivo y el propio usuario para valorar la adecuación de las acciones publicitarias generadas por los anunciantes. En definitiva, la adaptación a estos soportes digitales supone un reto y una oportunidad para el anunciante que en un futuro próximo es necesario valorar.

\section{Referencias bibliográficas}

BENAVIDES DELGADO, J. (et al.). (2010). «Los anunciantes españoles y el nuevo contexto de comunicación: una aproximación cualitativa», Revista Latina de Comunicación Social, $65,159-175$.

BudiU, R. \& NIELSEN, J. (2011). «Usability of iPad Apps and Websites» 2nd Edition, Publisher: Nielsen Norman Group, 1-129.

CORREDOR LANAS, P. (2011). «En clave digital: nuevos profesionales en Publicidad», Telos, 87, 97-100.

Costa, J. (2009). «Tabletas iPad de 5.300 años de edad», Pensar la Publicidad, vol. III (2), 209-214.

DEL PINO, C. (2007). «Nueva era en la comunicación comercial audiovisual: el marketing viral», Pensar la Publicidad, vol. I (2), 63-76.

De SALAS NESTARES, Ma . I. (2009). «La publicidad en las redes sociales: de lo invasivo a lo consentido», Icono $14,15,75-84$.

Distimo. http://www.distimo.com/ . Web visitada el 25 de febrero de 2012.

DOMINGO, G. \& MARTORELL, C. (2011). «Una mirada al consumidor de hoy (y de mañana) desde la publicidad», Trípodos, 28, 13-23.

FUNDACIÓN TELEFÓNICA (2011): Informe de la Sociedad de la Información en España 2010. Madrid, Telefónica. 
FUNDACIÓN TELEFÓNICA (2012): Informe de la Sociedad de la Información en España 2011. Madrid, Telefónica.

IAB SPAIN. III Estudio IAB Spain sobre Mobile Marketing. http://www.iabspain.net/ . Web visitada el 10 de diciembre de 2011.

IAB US. Tabvertising. iPad and other tablets: the advertising and marketing opportunities. http://www.iabspain.net/. Web visitada el 20 de marzo de 2012.

LEÓN SAEZ DE YBARRA, J. L. (2009). «Nuevos Soportes y mercados de la publicidad digital. Transiciones y experiencias», Pensar la Publicidad, vol. III (2), 17-30.

LÓPEZ, D. (2011). «El digital out of home (dooh) o digital signage, el otro marketing digital», Trípodos, 28, 25-34.

LÓPEZ DE AGUILETA CLEMENTE, C. \& TORRES ROMAY, E. (2007). «Medios y soportes alternativos para una publicidad convencional: publicidad "off the line"», Pensar la Publicidad, vol. I, (2), 117-130.

MOBILE MARKETING ASSOCIATION (2012). Libro blanco de las Apps. http://mmaspain.com/libro-blanco-apps/libro-3n.html. Web visitada el 5 de julio de 2012.

MARTÍ PARREÑO, J.M. (et al). (2011). Retos y oportunidades para incrementar la eficacia de la publicidad actual. Comunicación presentada en el III Congreso Internacional Latina de Comunicación Social en Tenerife.

PACHECO RUEDA, M. (Coord.) (2008): La publicidad en el contexto digital. Viejos retos y nuevas oportunidades, Sevilla, Comunicación social.

RosAles, P. (2010): Estrategia digital: cómo usar las nuevas tecnologías mejor que la competencia, Barcelona, Deusto.

Puro Marketing. http://www.puromarketing.com/12/11392/cada-tres-consumidores-lineautilizara-tablet-2014.html\%29. Web visitada el 25 de diciembre de 2011.

SÁNCHEZ REVILla, M. A. (2012): Resumen. Estudio Infoadex de la inversión de la comunicación publicitaria 2011, Madrid, Infoadex S.A., 11. http://www.infoadex.es/RESUMEN2011.pdf. Web visitada el 25 de febrero de 2012.

SolanA, D. (2010): Postpublicidad. Reflexiones sobre una nueva cultura publicitaria en la era digital, Barcelona, Doubleyou. 